\title{
Scissor-hinged retractable membrane structures
}

\section{Tom Van Mele*, Niels De Temmerman, Lars De Laet and Marijke Mollaert}

\author{
Department of Architectural Engineering, \\ Faculty of Engineering Sciences, \\ Vrije Universiteit Brussel, \\ Pleinlaan 2, 1050 Brussel, Belgium \\ Fax: +32(0)262928 41 \\ E-mail: tom.v.mele@gmail.com \\ E-mail: niels.de.temmerman@vub.ac.be \\ E-mail: lars.de.laet@vub.ac.be \\ E-mail: marijke.mollaert@vub.ac.be \\ *Corresponding author
}

\begin{abstract}
This paper introduces scissor-hinged retractable membrane structures, a system for retractable membrane roofs that require a fully retractable supporting structure and multiple stable roof-configurations. A vaulted, foldable, supporting structure is developed consisting of two scissor-hinged frames that can retract towards opposite sides of the space below. Structural membranes are spanned in these frames in a ridge-and-valley configuration to form the roof's outer surface. Actuators are integrated to control the tension in the membrane surface in different roof-configurations. Transformation from one configuration to another is controlled by cables running through the supporting structure over series of pulleys. The characteristics of these components are discussed, and their implementation illustrated with a design for a retractable roof over a tennis arena. The structural behaviour of this roof is analysed under representative load conditions. A procedure for such analyses using conventional software tools for the design and analysis of tensile surface structures is presented.
\end{abstract}

Keywords: retractable roofs; scissor mechanisms; membrane structures; artificial muscles.

Reference to this paper should be made as follows: Van Mele, T., De Temmerman, N., De Laet, L. and Mollaert, M. (2010) 'Scissor-hinged retractable membrane structures', Int. J. Structural Engineering, Vol. 1, Nos. 3/4, pp.374-396.

Biographical notes: Tom Van Mele received his $\mathrm{PhD}$ from the Vrije Universiteit Brussel, in 2008, on the topic of scissor-hinged retractable membrane structures. His professional interests include kinetic structures, form-finding and structural optimisation, and graphic analysis and design methods. Currently, he is involved in a project that aims to develop a comprehensive e-learning environment for structural analysis and design based on interactive statics.

Niels De Temmerman received his $\mathrm{PhD}$ in 2007 on the topic of adaptable structures for architectural applications. His research topics include lightweight structures, tensile surface structures, deployable structures, 4D-design and computer aided design. He is currently teaching architectural design studio and parametric modelling and is a member of AE-lab, the Research Group for Architectural Engineering. 
Lars De Laet is currently a PhD Researcher, conducting research on deployable Tensairity structures with funding of the research foundation - Flanders (FWO). He received his Masters in Architectural Engineering in 2006 on the topic of scissor hinged structures. He is also a member of AE-lab, the Research Group for Architectural Engineering.

Marijke Mollaert received her PhD in 1984. She worked at Samyn and Partners from 1985 till 1987 and in the Structural Analysis Department of Portal Lessines SA from 1991 till 1993. She is a Professor at the VUB since 2000, teaching computer aided design, tensile surface structures and building physics. She is the Executive Secretary of the TensiNet Association since 2004. Her research topics include tensile surface structures, deployable structures and textile formwork for concrete structures. She is a member of AE-lab, the Research Group for Architectural Engineering.

\section{Introduction}

\subsection{Retractable membrane roofs}

Retractable roofs are roof structures that can transform from one configuration to another, providing variable cover to the space below (Jensen, 2004; Ishii, 2000).

Retractable membrane roofs form a specific category of retractable roof structures, because their roof surface is formed by a tensioned membrane. Membranes are very suitable for the outer skin of transformable constructions; they are light and flexible, and, therefore, they can be easily transformed from a compact bunched or folded configuration to a much larger surface. However, because these membranes have to be tensioned in a specific shape in order to carry external loads, retractable membrane structures can often only be used in one configuration, typically the closed roof. Furthermore, in some cases, retractable membrane roofs require a permanent supporting structure that remains over the space below, even when the roof is open.

Therefore, in this paper, a new solution is presented for retractable membrane roofs with a fully retractable supporting structure and multiple stable roof-configurations.

\subsection{Scissor-hinged membrane structures}

With a series of parallel linear scissor mechanisms, interconnected by simple pin-jointed struts, a vaulted, foldable frame can be assembled. Two of these frames, each with one side connected to the ground and the other cantilevered over an additional rotating structure, form the basis of the retractable supporting structure of the roof. Both halves of this supporting structure can retract individually or simultaneously towards opposite sides of the space below, or unfold and connect in the middle to form a closed roof.

The transformation of the roof from one configuration to another and its stabilisation in a load-carrying configuration is controlled by a system of cables running through the scissor mechanisms over series of pulleys. One set of cables is used to retract the structure, the other to unfold it.

The roof's surface consists of tensioned structural membranes. These membranes span between the parallel scissor mechanisms of each of the two collapsible roof frames in a ridge-and-valley configuration; the ridge cables are connected to the top hinges of the 
scissors, and the valleys to the bottom hinges. During transformation of the roof, the membrane surface folds or unfolds together with the scissors while preserving its overall wave-like shape.

Actuators are integrated to control the tension in the roof's membrane surface, which not only facilitates the transformation process, but also allows the roof to be used in multiple configurations.

\subsection{The roof of a tennis arena}

We will discuss the different components of the roof, starting with the foldable supporting structure, and illustrate how they fit in the entire system by using them in a roof for a medium span sports facility (Figure 1). We have used the dimensions of a tennis arena as an example. Although tennis is both an indoor and outdoor sport, it can only be played in good weather conditions. A tennis arena is, therefore, a good example of a venue that benefits greatly from having a retractable roof.

Figure 1 A scissor-hinged retractable membrane roof for a tennis arena (see online version for colours)

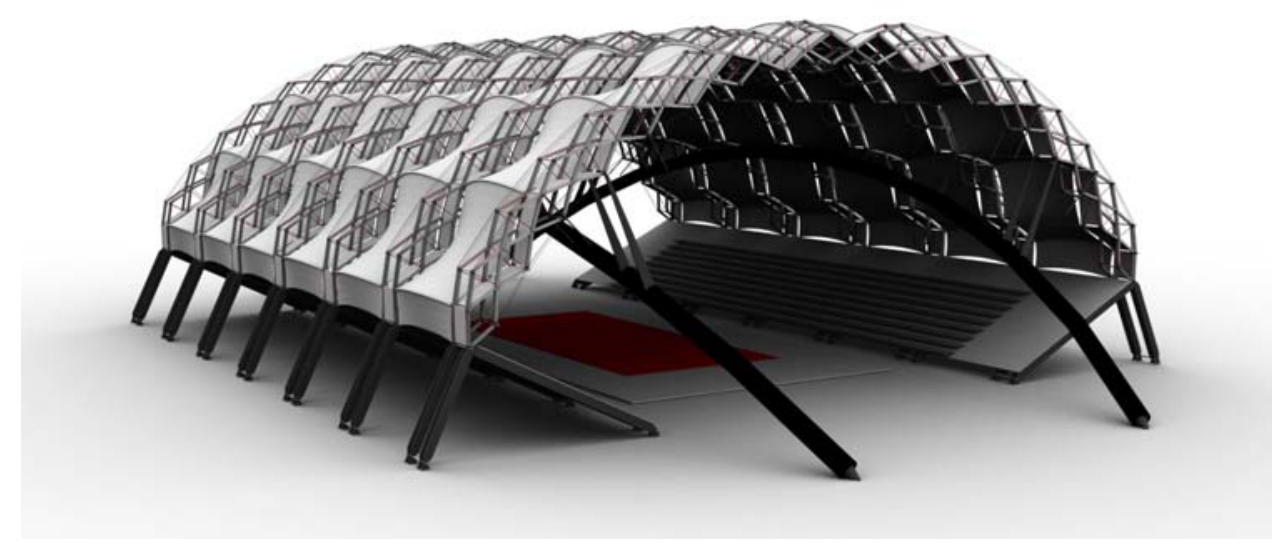

We have chosen the orientation of the roof so that the opening and closing direction is perpendicular to the long side of the court. This way, the individual roof panels can cover the spectator area without covering the playing field. In the closed configuration, when the panels are connected to each other in the middle, the roof spans approximately $44 \mathrm{~m}$.

\section{Scissor-hinged supporting structure}

Scissor units consist of two bars connected by a revolute joint. This joint, or scissor hinge, allows the bars to rotate around an axis perpendicular to their common plane. Altering the location of the scissor hinge or the shape of the bars gives rise to three distinct unit types: translational, polar and angulated (Figure 2). When series of scissor units are interconnected at the ends of the bars by revolute joints, linear or surface-like scissor mechanisms can be formed (De Temmerman, 2007). 
Figure 2 Three types of basic scissor units: (a) translational (b) polar (c) angulated

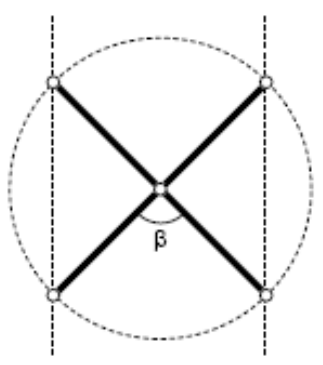

(a)

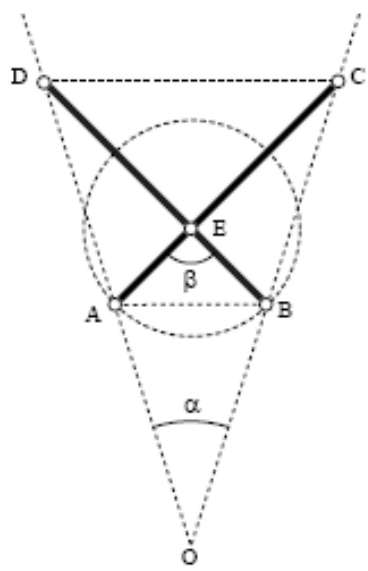

(b)

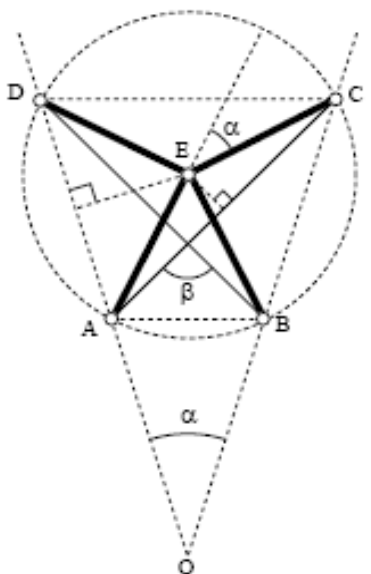

(c)

Figure 3 A ridge-and-valley membrane surface spanned between parallel linear scissor mechanisms
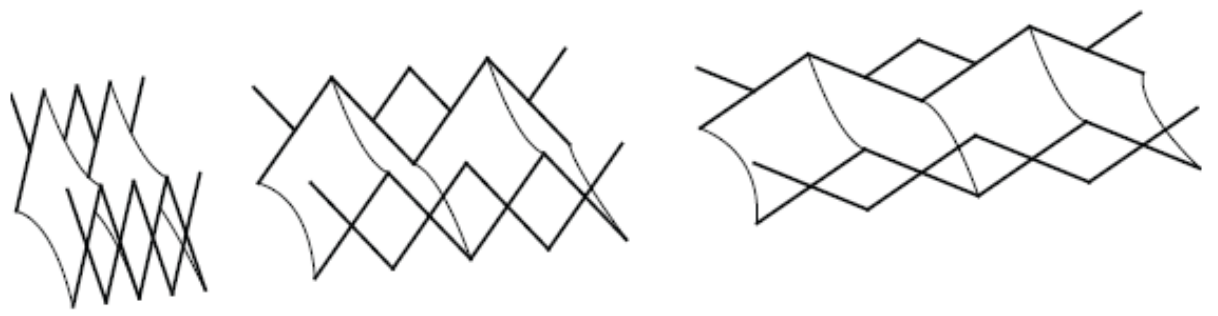

Note: The membrane folds with the scissors in a wave-like configuration.

Countless variations of shapes are possible, but, generally speaking, the more exotic the mechanisms become, the less useful the resulting structures are as foldable space enclosures. Therefore, we have only considered basic mechanisms, with regular geometry, consisting of identical bars and identical units.

From a series of parallel, linear scissor mechanisms, interconnected by simple pinjointed struts, a foldable frame can be assembled. Membranes can be spanned in such a frame, in a classic ridge-and-valley configuration. During transformation, the scissorhinged frame and the membrane fold or unfold together while preserving the wave-like shape of the surface (Figure 3).

The frames can be formed with all three types of linear mechanisms. However, since the presented system is intended for applications of relatively large span $( \pm 50 \mathrm{~m})$, only curved mechanisms are investigated; they are structurally more efficient and provide more interesting spatial solutions. Basic translational mechanisms are straight, whereas basic polar and angulated mechanisms are curved. 


\subsection{Unfolded configuration}

The design of the scissor mechanism for this retractable structure is based on the geometry of the closed roof. In this configuration the scissor mechanism is in its most unfolded state, where it is required to have a specific span, $S$, and leave a space underneath with specific height, $H$. The unfolded configuration of the mechanism can be characterised by the angle between the scissor bars, $\beta_{\text {uf }}$. Typically, span and height in the unfolded configuration are given; $\beta_{\mathrm{uf}}$ and the number of units in the mechanism are chosen; and the geometry of the bars is to be determined.

\subsubsection{Polar mechanisms}

A polar mechanism with $n$ units describes, with its scissor hinges, a circular curve that defines a sector of a circle with chord $S$. At midspan, the height of the curve is $H$, as seen in Figure 4. With $S$ and $H$ given, the radius, $R$, of the circle and the central angle, $\gamma$, of the sector can be determined using basic trigonometry:

$$
\begin{aligned}
& R=\frac{S^{2}}{8 H}+\frac{H}{2} \\
& \gamma=2 \operatorname{ArcSin}\left[\frac{S}{2 R}\right]
\end{aligned}
$$

Figure 4 A basic polar mechanism defines a sector of a circle with central angle $\gamma$ and radius $R$

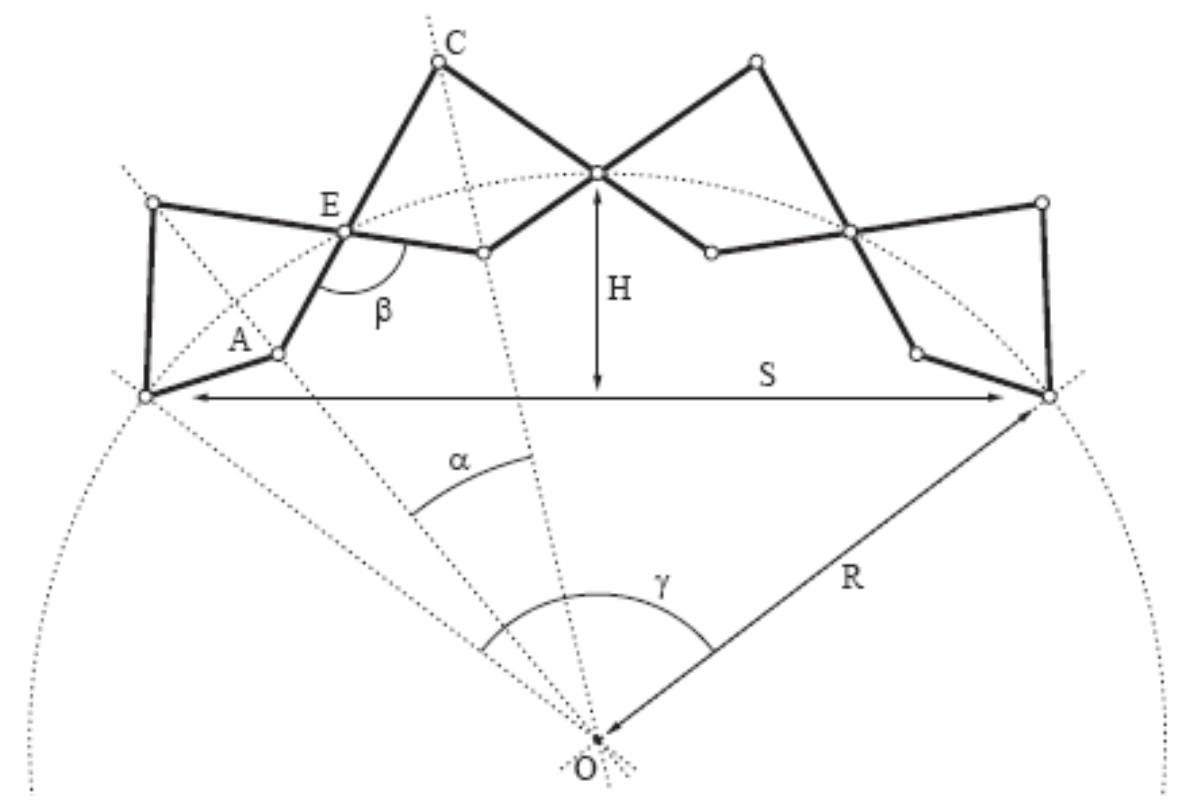


Therefore, using the $\mathrm{n}$-fold symmetry of the mechanism, each unit defines a subsector with central angle $\alpha=\gamma / n$. From $\alpha, R$, and $\beta_{\text {uf }}$, we can determine the geometry of the individual bars of a polar mechanism with (Van Mele, 2008):

$$
\begin{aligned}
& (\overline{A E})_{\mathrm{pol}}=\frac{R \cdot \operatorname{Tan}[\alpha / 2]}{\operatorname{Sin}\left[\beta_{\mathrm{uf}} / 2\right]+\operatorname{Cos}\left[\beta_{\mathrm{uf}} / 2\right] \cdot \operatorname{Tan}[\alpha / 2]} \\
& (\overline{C E})_{\mathrm{pol}}=\frac{R \cdot \operatorname{Tan}[\alpha / 2]}{\operatorname{Sin}\left[\beta_{\mathrm{uf}} / 2\right]-\operatorname{Cos}\left[\beta_{\mathrm{uf}} / 2\right] \cdot \operatorname{Tan}[\alpha / 2]}
\end{aligned}
$$

\subsubsection{Angulated mechanisms}

The geometry of the elements of an angulated unit is related to the geometry of the elements of a polar unit, as depicted in Figure 2. Therefore, the length of the rigidly connected segments of the bars of an angulated unit can be derived from the length of the bars of a polar unit and the angle $\alpha$ :

$$
(\overline{A E})_{\mathrm{ang}}=\frac{(\overline{A E}+\overline{C E})_{\mathrm{pol}}}{2 \operatorname{Cos}[\alpha / 2]}
$$

Figure 5 An angulated mechanism can be derived from a polar mechanism

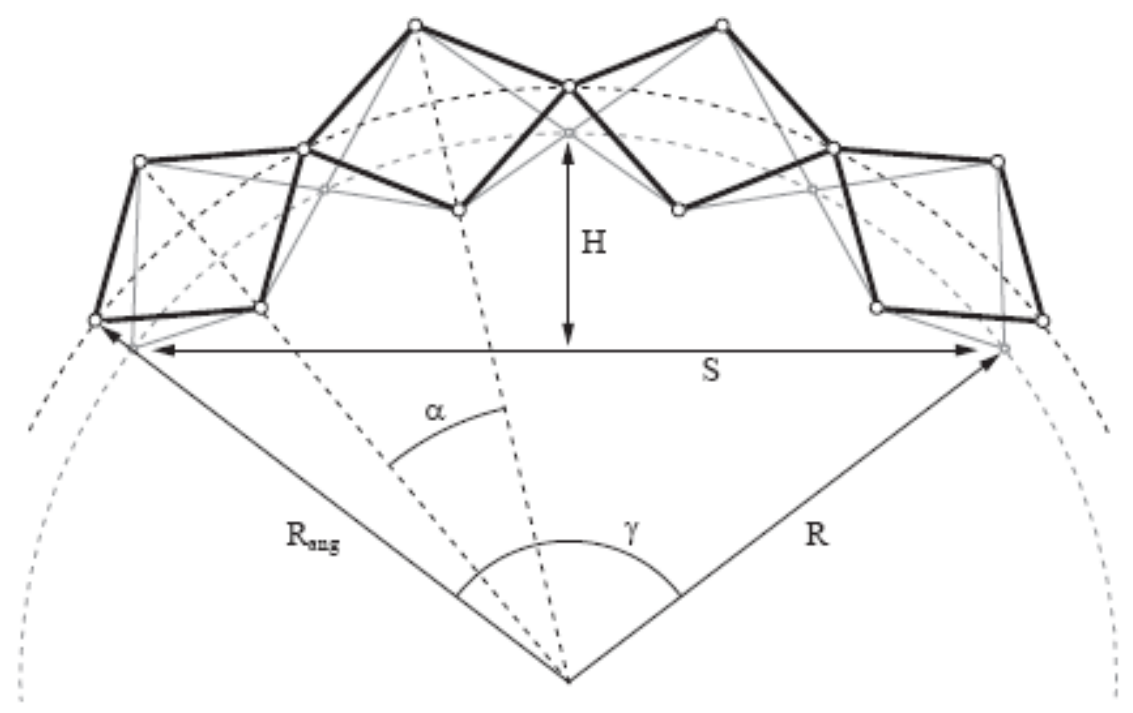

Note: In order for their span and height to match, the angulated mechanism should be scaled by a factor $\psi=R / R_{\text {ang }}$.

The circle with radius $R_{\text {ang }}$, described by the intermediate hinges of the angulated structure, is bigger than the circle with radius $R$, described by the intermediate hinges of the polar structure, as seen in Figure 5. For the span and height of the angulated mechanism to match the required span and height of the roof in the unfolded configuration, the elements of the angulated mechanism should be scaled by a factor $\psi=R / R_{\text {ang }}$, with (Van Mele, 2008): 


$$
R_{\mathrm{ang}}=R+(\overline{A E})_{\mathrm{ang}} \cdot \operatorname{Cos}\left[\frac{\beta_{\mathrm{uf}}-\alpha}{2}\right]-(\overline{A E})_{\mathrm{pol}} \cdot \operatorname{Cos}\left[\frac{\beta_{\mathrm{uf}}}{2}\right]
$$

\subsection{Transformation process}

By varying $\beta$, a scissor mechanism folds or unfolds. The behaviour of polar and angulated mechanisms during transformation is completely different, even if they have the same geometry in the unfolded configuration at $\beta_{\mathrm{uf}}$. This follows immediately from equation (5), which is the relationship between $\alpha$ and $\beta$ in polar and angulated units at any stage of the transformation process (You and Pellegrino, 1997):

$$
\operatorname{Tan}\left[\frac{\alpha}{2}\right]=\frac{\overline{C E}-\overline{A E}}{\overline{A C}} \cdot \operatorname{Tan}\left[\frac{\beta}{2}\right]+\frac{\overline{E F}}{\overline{A F}}
$$

In a polar unit, $\overline{E F} / \overline{A F}=0$. Therefore, in every configuration, $\alpha$ is directly proportionate to $\beta$. The ratio of $\alpha$ to $\beta$ is constant for a specific unit, and depends on the location of the scissor hinge:

$$
\alpha_{\beta}=\phi \cdot \beta \text { with } \phi=\operatorname{ArcTan}\left[\frac{\overline{C E}-\overline{A E}}{\overline{A C}}\right]
$$

In an angulated unit, on the other hand, the two rigidly connected segments of each angulated bar are of equal length, $C E=A E$ (Figure 2). Therefore, in an angulated mechanism, $\alpha$ is independent of $\beta$ :

$$
\alpha_{\beta}=\alpha=2 \operatorname{ArcTan}\left[\frac{E F}{A F}\right]
$$

In any configuration, corresponding to a specific value of $\beta$, the scissor hinges of a polar scissor mechanism lie on a circle with radius $\left(R_{\beta}\right)_{\text {pol }}$, whereas those of an angulated mechanism - after scaling - lie on a circle with radius $\left(R_{\beta}\right)$ ang (Van Mele, 2008):

$$
\begin{aligned}
\left(R_{\beta}\right)_{\mathrm{pol}} & =(\overline{A E})_{\mathrm{pol}} \cdot\left(\frac{\operatorname{Sin}[\beta / 2]}{\operatorname{Tan}[\phi \cdot \beta / 2]}+\operatorname{Cos}\left[\frac{\beta}{2}\right]\right) \\
\left(R_{\beta}\right)_{\mathrm{ang}} & =\psi \cdot(\overline{A E})_{\mathrm{ang}} \cdot\left(\frac{\operatorname{Sin}[(\beta-\alpha) / 2]}{\operatorname{Tan}[\alpha / 2]}+\operatorname{Cos}\left[\frac{\beta-\alpha}{2}\right]\right)
\end{aligned}
$$

With $\gamma_{\beta}=n \cdot \phi \cdot \beta$ for a polar mechanism and $\gamma_{\beta}=n \alpha$ for an angulated one, span and height in configuration $\beta$ can be determined with:

$$
\begin{aligned}
& S_{\beta}=2 R_{\beta} \cdot \operatorname{Sin}\left[\frac{\gamma_{\beta}}{2}\right] \\
& H_{\beta}=R_{\beta}\left(1-\operatorname{Cos}\left[\frac{\gamma_{\beta}}{2}\right]\right)
\end{aligned}
$$

The transformation process of a polar and an angulated mechanism is depicted in Figure 6. 
Figure 6 Transformation processes of a polar (top) and an angulated mechanism (bottom), each with 10 units

$$
\beta=0 \quad \beta=30 \quad \beta=60 \quad \beta=90 \quad \beta=120
$$
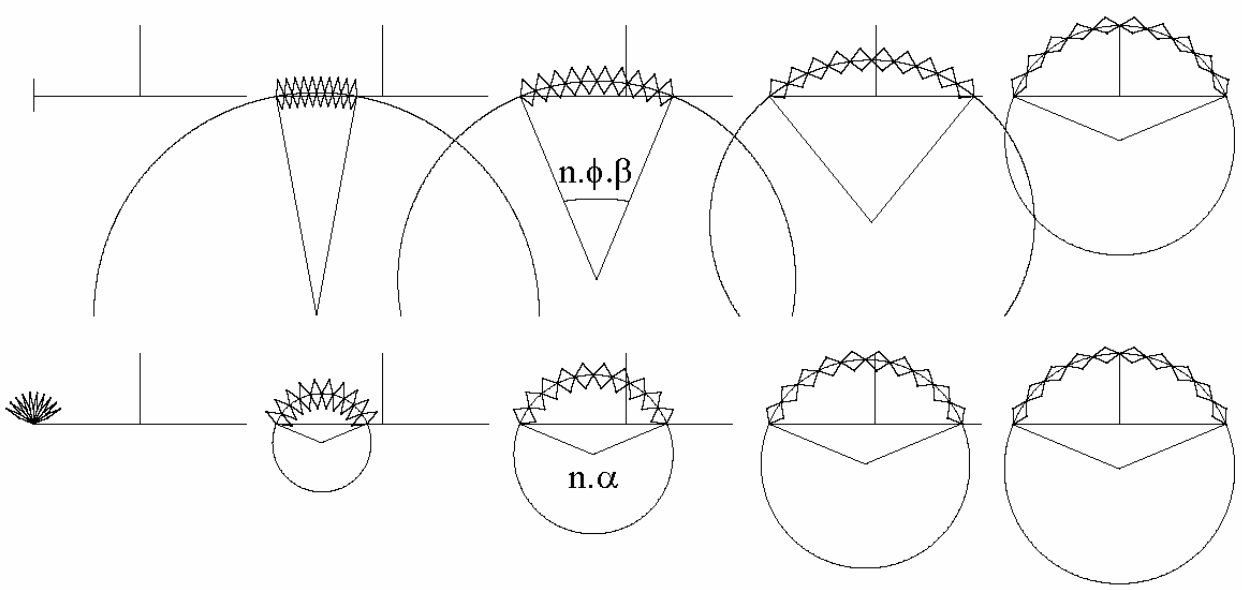

Note that the polar mechanism becomes more and more and more curved as it unfolds. At some point it reaches its maximum span, and then it curls up until it forms a circle. The behaviour of the angulated mechanism is completely different. Since $\gamma$ is independent of $\beta$, the mechanism has the same proportions in every configuration.

\subsection{Multi-angulated bars}

An important characteristic of angulated mechanisms is that they can form a curved, foldable lattice consisting of rigid multi-angulated bars (Jensen, 2004). These lattices cannot only be used to improve the structural behaviour of the roof's supporting structure, as we will see in Section 5, they also provide a way to create a fully closed roof surface, which will be described in Section 4.

In the unfolded configuration, the hinges of a multi-angulated structure lie on concentric circles with radii $R_{\text {ang }}^{i}$, with $i=0,1,2,3 \ldots$ (Figure 7 ). The radius of the circle described by the scissor hinges of the original mechanism is $R_{\text {ang }}^{0}$ and can be calculated with equation (4). The radii of the more outward circles, $R_{\mathrm{ang}}^{+i}$ and of the more inward circles, $R_{\text {ang }}^{-i}$ can be found with (Van Mele, 2008):

$$
\begin{aligned}
& R_{\mathrm{ang}}^{i}=R_{\mathrm{ang}}^{i-1} \cdot \operatorname{Cos}\left[\frac{\alpha}{2}\right]+\psi \cdot(\overline{A E})_{\mathrm{ang}} \cdot \operatorname{Cos}\left[\frac{\beta_{\mathrm{uf}}+(i-1) \alpha}{2}\right] \\
& R_{\mathrm{ang}}^{-i}=R_{\mathrm{ang}}^{-i+1} \cdot \operatorname{Cos}\left[\frac{\alpha}{2}\right]-\psi \cdot(\overline{A E})_{\mathrm{ang}} \cdot \operatorname{Cos}\left[\frac{\beta_{\mathrm{uf}}+(1-i) \alpha}{2}\right]
\end{aligned}
$$

During transformation, a multi-angulated mechanism behaves in the same way as a regular angulated mechanism, but in the folded configuration it is considerably larger than one with regular angulated bars, as seen in Figure 10. 
Figure 7 With angulated mechanisms, curved foldable lattices can be formed, consisting of multi-angulated bars

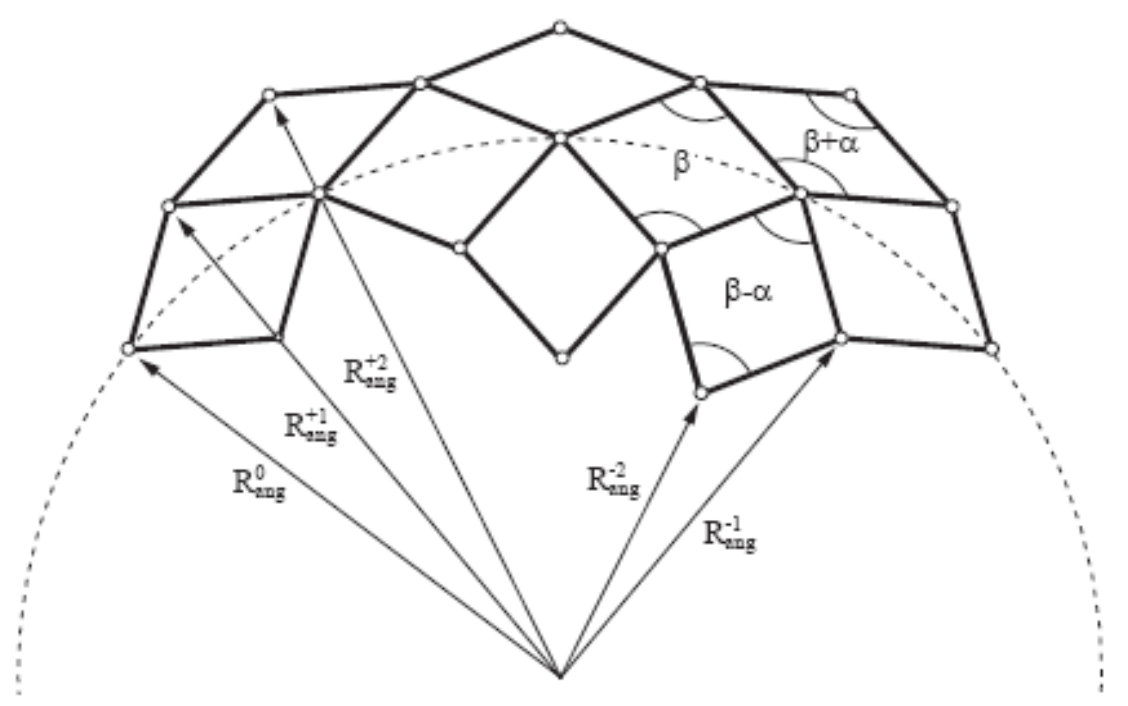

\subsection{Arena roof-supporting structure}

The roof for the tennis arena consists of two scissor-hinged frames that can retract to opposite sides of the space below. Figure 8 depicts the movement of the frames over a series of additional supporting arches.

To avoid such a permanent supporting structure that remains over the space below, even when the roof is open, each of the frames can be cantilevered over an additional supporting structure that rotates with the frame during transformation, as shown in Figure 9.

Note that a polar mechanism should be designed to reach its final configuration before or exactly at maximum span. Otherwise the roof won't be able to close, because the frames would have to overlap before reaching their final position. This problem does not occur with an angulated mechanism.

Figure 8 The two scissor-hinged frames of the roof of a tennis arena can be supported by a series of permanent arches; on the left, a polar mechanism, and on the right, an angulated one

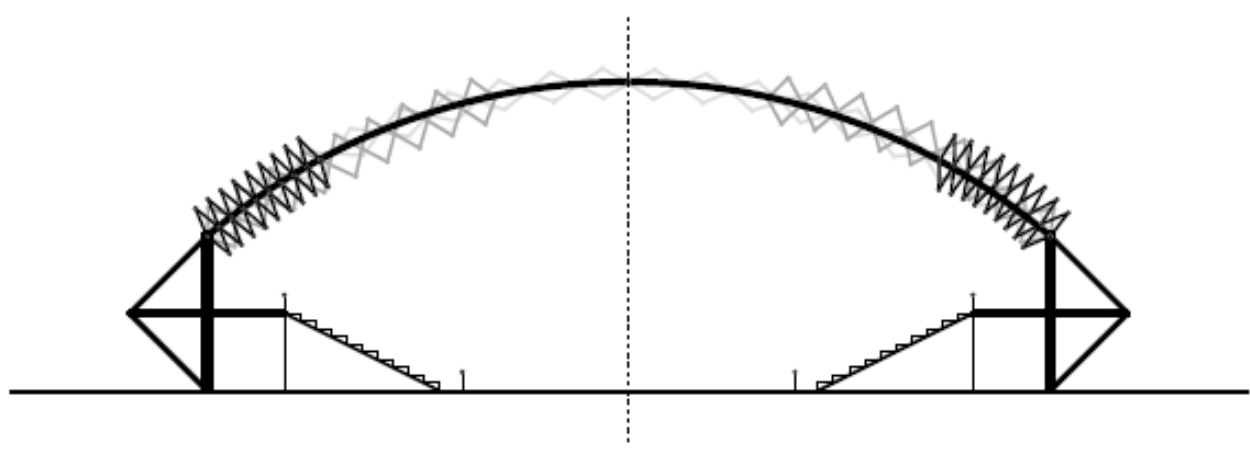


Figure 9 To avoid having a permanent supporting structure over the space below, even when the roof is open, a rotating frame can be used to support the two frames of the roof; on the left, a polar mechanism, and on the right, an angulated one

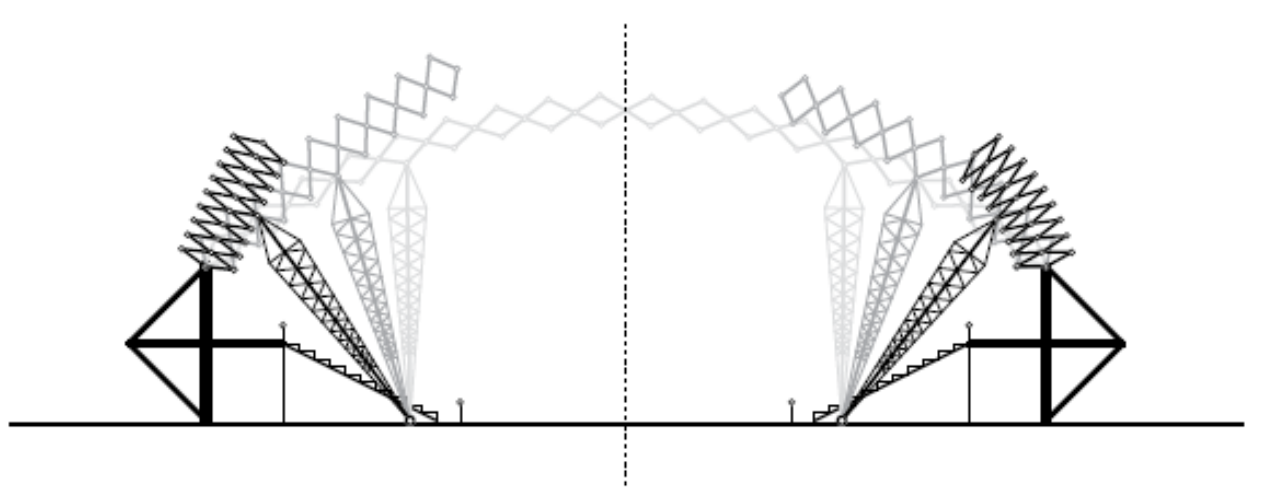

Figure 10 In the folded configuration, a multi-angulated mechanism is considerably larger than a mechanism with regular angulated bars

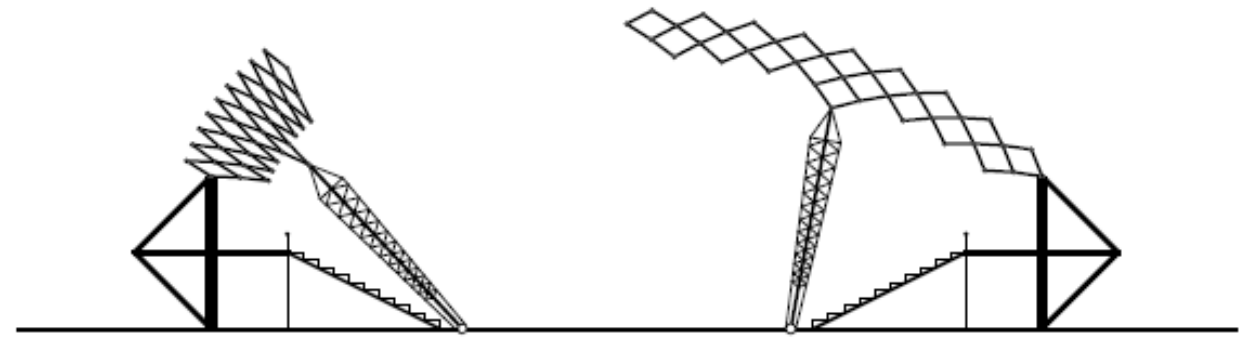

To provide insight in the design, behaviour, and characteristics of scissor mechanisms in general, and to get more familiar with the design of scissor mechanisms for retractable roofs as discussed in this paper, we have created a series of web-based interactive tools that are available at http://www.tomvanmele.net/interactive.html.

\section{Opening and closing - transformation control}

In configurations in which they are required to carry loads, scissor mechanisms have to be restrained to prevent them from folding or unfolding under influence of the applied loads. Elements that can transform the mechanisms from one load-carrying configuration to another should also be integrated. Here, we will discuss how cables can be used to accomplish both of these aspects of transformation control.

\subsection{Individual cable segments}

A linear scissor mechanism has only one degree of freedom, regardless of the number of units it contains. Therefore, strictly speaking, only two cables are required to transform a scissor mechanism into a statically determinate structure under any given load. Additional cables are redundant, but could be used to increase the safety of the structure 
and to improve its structural performance. The cables can be rolled onto a spool by a motor to transform the structure from one configuration to another.

Figure 11 Individual cable segments may be used to control the transformation of the roof from one configuration to another, and rigidify the mechanism in configurations where it is required to carry loads (see online version for colours)

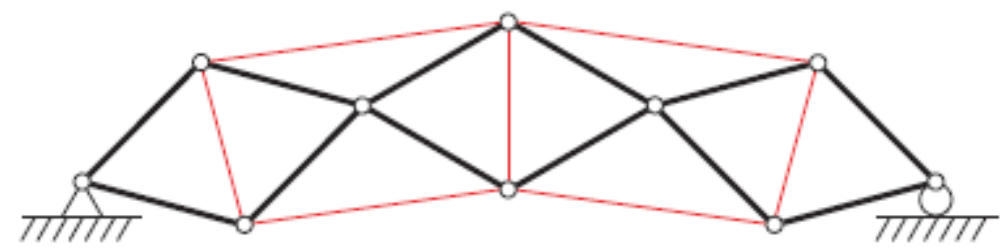

There are some practical considerations to a setup with individually controlled cable segments (Figure 11). For example, a separate spool and motor are required for each of the cable segments, which adds considerable weight to the structure, especially if the mechanism contains a high number of units; and the actuation of the individual cable segments should be synchronised to avoid a build up of stresses in the bars during transformation.

An interesting alternative is a cable-pulley system. In a cable-pulley system, the individual cable segments are replaced by a continuous cables running over series of pulleys. The advantage of this system is that, regardless of the number of units in the mechanism, only three cables have to be used to achieve a situation similar to the one depicted in Figure 11; and the spools onto which these cables have to be rolled to control the transformation can be placed outside the structure's span.

\subsection{Unfolding cable}

The 'vertical cables' in Figure 11 - the cables between a top and a bottom hinge - can be replaced by one continuous cable. This cable starts at the left, coming from the spool outside the structure's span, and zigzags through the structure over 12 pulleys to reach the other side, as seen in Figure 12. If the cable is rolled onto the spool, the mechanism unfolds.

Figure 12 A scissor mechanism can be unfolded with one cable running over a series of pulleys (see online version for colours)

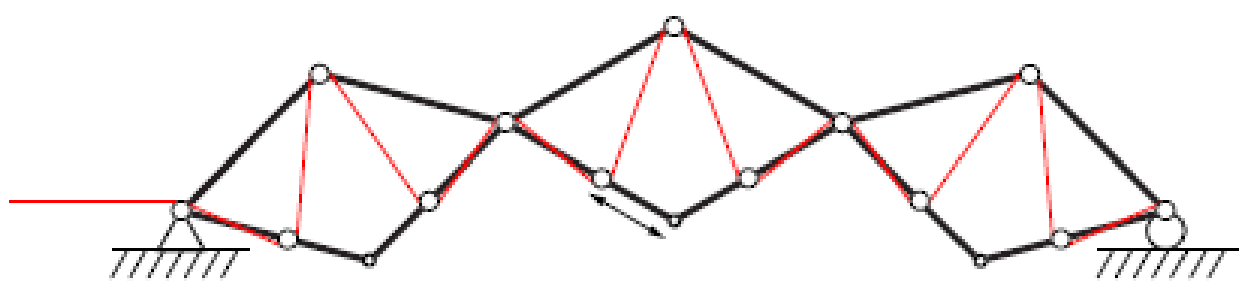

Note: If the cable is rolled onto a spool, the mechanism unfolds. 
The location of the bottom pulleys can be chosen anywhere on the lower segments of the angulated scissor bars. Their position influences the required cable stroke and the flow of forces through the system.

\subsection{Folding/retracting cable}

The 'horizontal cables' in Figure 11 - the cables between two top hinges or two bottom hinges - can also be replaced by continuous cables: one cable for the top segments (Figure 13) and one for the segments at the bottom (Figure 14).

In both cases, the cable starts at the left, at the spool outside the structure's span, and runs over eight pulleys to reach the other side.

Figure 13 A scissor mechanism can be folded with one cable running over a series of pulleys (see online version for colours)

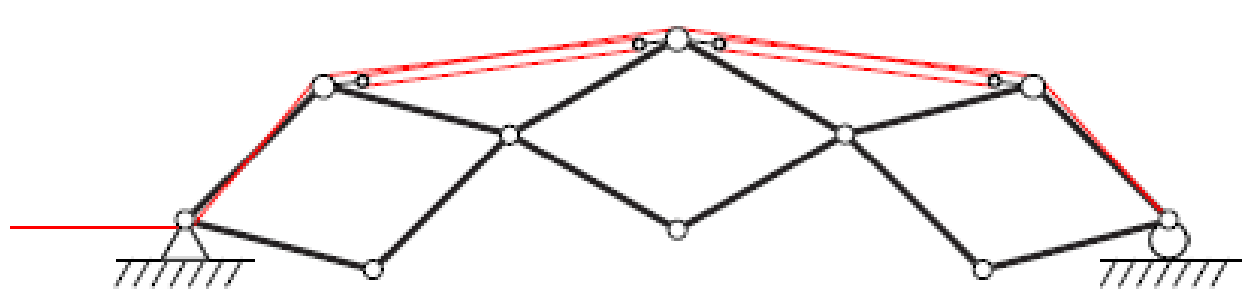

Note: If the cable is rolled onto a spool, the mechanism folds.

Figure 14 A scissor mechanism can be folded with one cable running over a series of pulleys (see online version for colours)

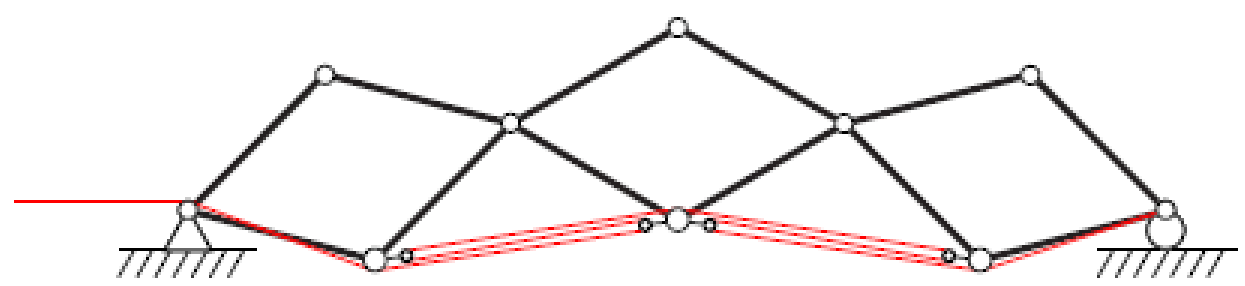

Note: If the cable is rolled onto a spool, the mechanism folds.

\subsection{Arena roof-transformation control}

Figure 15 shows how the folding and unfolding cables are integrated in the supporting structure to control the transformation of the roof. Note that in the depicted configuration (the unfolded frame), the unfolding cable is rolled completely onto a spool, and that the top and bottom folding cables are completely unrolled. 
Figure 15 One frame of the roof of a tennis arena with the cable-pulley system; the unfolding cable, and the two folding cables

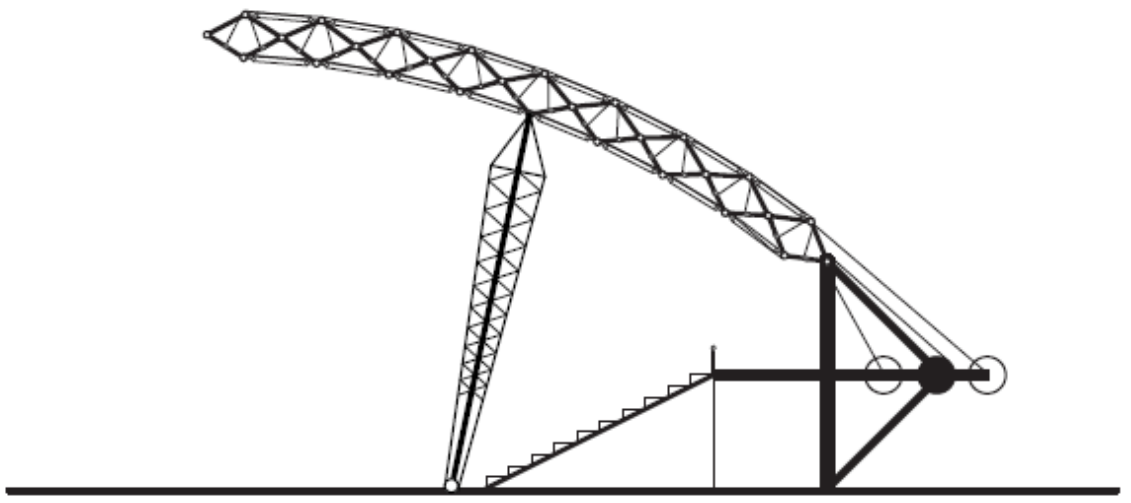

\section{Roof surface}

The surface of the retractable roof is formed with structural membranes. The membranes are spanned between the parallel scissor arches in a ridge-and-valley configuration; the ridge cables are connected to the top hinges of the scissors and the valley cables to the bottom hinges. This way, the membrane surface can fold and unfold with the scissors while preserving its wave-like shape.

Figure 16 The membranes are spanned between the parallel scissor arches in a ridge-and-valley configuration

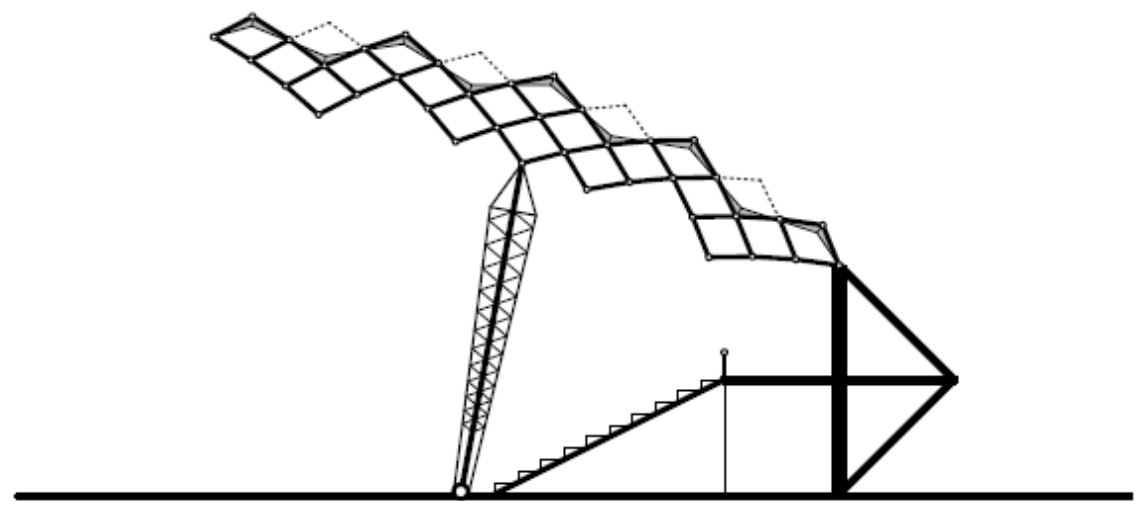

Note: In a multi-angulated mechanism, some of the bar segments can be left out.

\subsection{Actuation}

The shape of a tensioned membrane surface is related to the geometry and characteristics of its boundaries and internal cables, and the level of mechanically applied (pre-)stress. Since in a retractable roof, the configuration of the boundaries and internal cables changes from one configuration to another, the membrane can only be correctly tensioned 
in one configuration: the configuration for which its original doubly curved shape was determined. In every other configuration, the membrane will be stretched or hang loose.

This is also the case for a ridge-and-valley surface. Considering that a ridge-and-valley surface has only slightly warped panels, the main reason for this is the curvature of the internal cables that serve as foldlines during the transformation process. Because of this curvature, the distance between the foldlines changes during transformation, which results in the membrane hanging loose or being stretched.

Figure 17 A ridge-and-valley surface with two ridges and one valley, spanned in a small scissor-hinged frame; viewed from the side

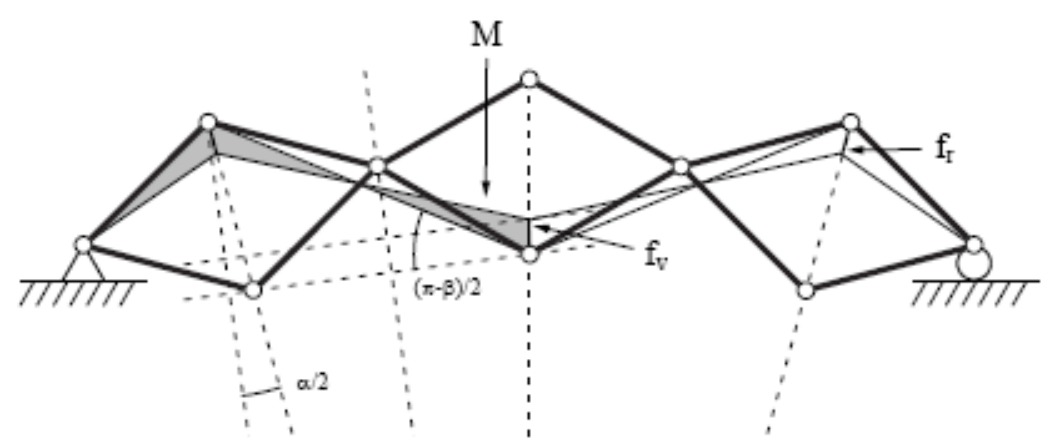

The distance between the ridges and the valleys at $\operatorname{midspan}^{2}, M$, as seen in Figure 17, can be calculated from the geometry of the scissor bars, the sag of the ridge and valley cables at midspan, $f_{\mathrm{r}}$ and $f_{\mathrm{v}}$, and the angle $\beta$ (Van Mele, 2008):

$$
\begin{aligned}
M^{2} & =\left[\left(2(\overline{A E})_{\mathrm{ang}} \cdot \operatorname{Cos}\left[\frac{\alpha}{2}\right]\right) \cdot \operatorname{Cos}\left[\frac{\beta}{2}\right]-\operatorname{Cos}\left[\frac{\alpha}{2}\right]\left(f_{r}+f_{v}\right)\right]^{2} \\
& +\left[\left(2(\overline{A E})_{\mathrm{ang}} \cdot \operatorname{Cos}\left[\frac{\alpha}{2}\right]\right) \cdot \operatorname{Sin}\left[\frac{\beta}{2}\right]-\operatorname{Sin}\left[\frac{\alpha}{2}\right]\left(f_{r}-f_{v}\right)\right]^{2}
\end{aligned}
$$

With $(\overline{A E})_{\text {ang }}, \alpha, f_{\mathrm{r}}$, and $f_{\mathrm{v}}$ constants, equation (11) can be rewritten to:

$$
M^{2}=\left(C_{1} \cdot \operatorname{Cos}\left[\frac{\beta}{2}\right]-C_{2}\right)^{2}+\left(C_{1} \cdot \operatorname{Sin}\left[\frac{\beta}{2}\right]-C_{3}\right)^{2}
$$

In the case where $f_{\mathrm{r}}=f_{\mathrm{v}} \Rightarrow C_{3}=0$, this can be simplified to:

$$
M=\sqrt{C_{1}^{2}-2 C_{1} C_{2} \cdot \operatorname{Cos}\left[\frac{\beta}{2}\right]}+C_{2}^{2}
$$

Since $C_{1} \gg C_{2}, M$ will be higher for high values of $\beta$, and smaller for small values of $\beta$. Therefore, if the original surface is determined in the unfolded configuration, the membrane will hang loose in more folded configurations, and vice versa. 


\subsection{Pleated pneumatic artificial muscles}

In order to control the tension in the membrane surface, we have added pleated pneumatic artificial muscles (PPAM). PPAM are air-pressured actuators that contract when inflated (Daerden and Lefeber, 2002) (Figure 18).

Figure 18 PPAM are air-pressured artificial muscles that contract when they are inflated

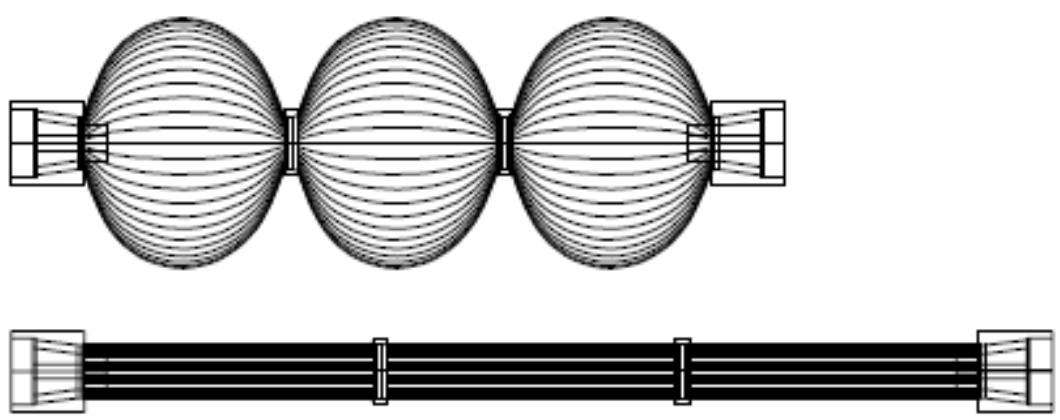

Some changes have to be made to the supporting structure to facilitate the integration of these actuators (Figure 19). First, each scissor arch is doubled. The original scissor arch and its double are connected with bars that serve as pins for the top and bottom hinges. Bracing cables prevent shearing of the two scissor arches relative to each other. To recreate a closed roof surface, panels are added on top of the scissors, following the same wave-like pattern as the membrane surface [Figures 19(c) and 19(d)]. The ridge cables are connected to the top hinges, as before, but the valley cables continue through the doubled arches [Figure 19(d)].

Note that the scissor bars of each of the doubled arches are also organised in separate layers to facilitate the integration of the cable-pulley system and to avoid interference of the bars. The resulting openings in the roof's surface can be avoided using a multi-angulated mechanism. In a multi-angulated mechanism, the segments of the bars that 'penetrate' the roof's surface can be omitted without affecting the kinematics of the mechanism (Figure 16).

Finally, two PPAM per valley cable are added to the structure. One side of each of these actuators is connected to the valley cables, the other to the adjacent top hinges, as depicted in Figure 19. The idea is that the PPAM can control the tension in the membrane surface by pulling the valley cables more or less into the scissor arches, because this influences the curvature of the valley cables and therefore the value of $M$. This means that, for example, the PPAM can be used to loosen the membranes before each transformation of the roof to a different configuration, and then re-tension the membranes after the new configuration is reached, which facilitates the action of the cable-pulley system during the transformation process.

We have tested this system on a small module of a ridge-and-valley surface (Block and Van Mele, 2003). The module is supported by foldable triangles mounted on steal beams that can slide over the ground to fold and unfold the surface. The configuration of the PPAM and the valley cables is slightly different, but the principle is the same (see Figure 20). The PPAM could sufficiently control the tension in the membrane in 
configurations between $\beta_{\mathrm{f}}=30^{\circ}$ and $\beta_{\mathrm{uf}}=120^{\circ}$ (Figure 20). Note that these results were only evaluated visually; the tension in the membrane was not actually measured.

Figure 19 Some changes had to be made to the supporting structure to facilitate the integration of the PPAM actuators (see online version for colours)
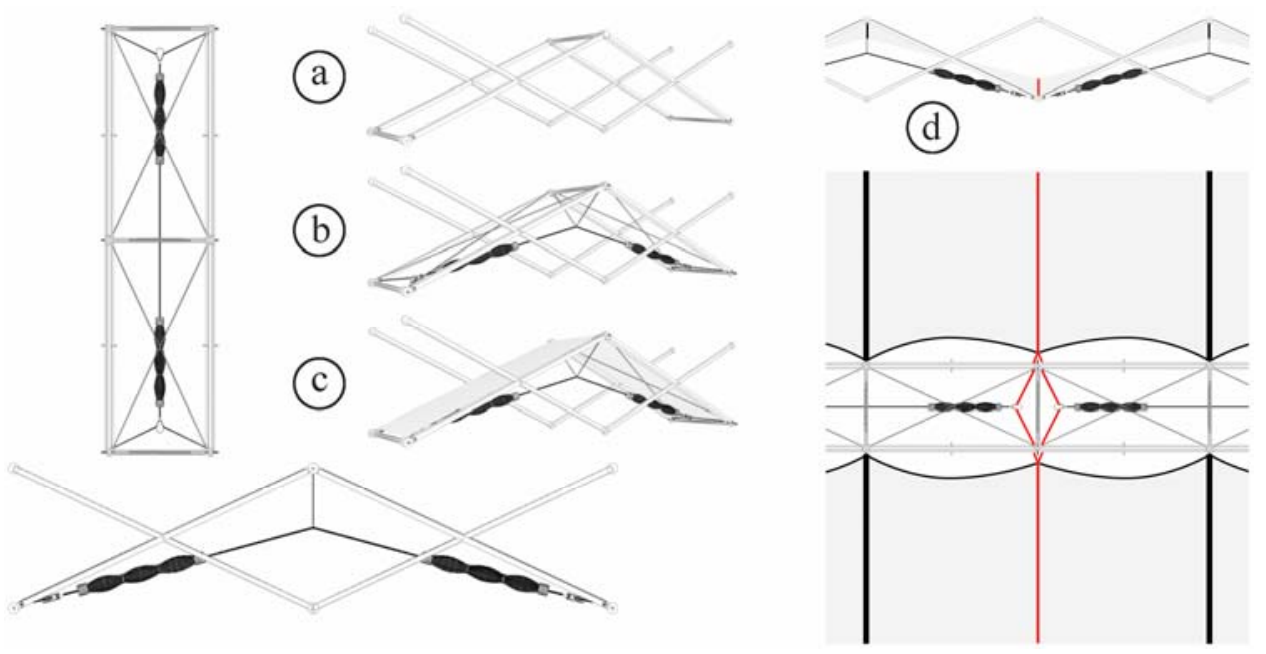

Notes: PPAM are integrated in the space between the doubled scissor arches. There, they can pull the valley cables to control the tension in the membranes. The cable-pulley system is not included in this picture.

Figure 20 The principle of controlling membrane tension of a ridge-and-valley surface in several configurations with PPAM was tested on a small module

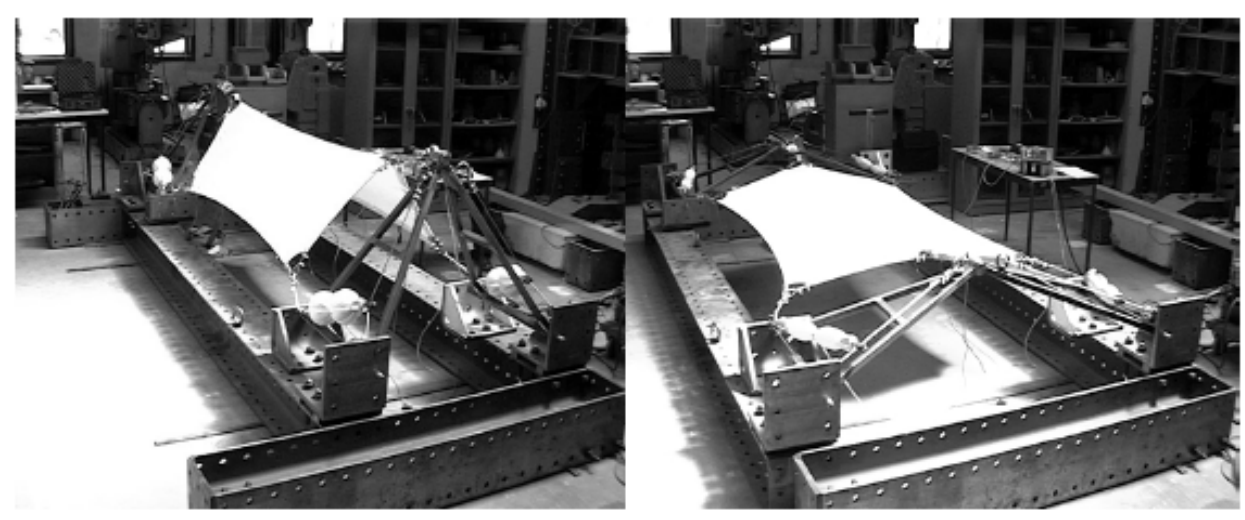

\section{Structural behaviour}

Some tentative calculations were performed on the roof of the tennis arena, as discussed in Subsection 1.3. The goal of these calculations was to assess the structural feasibility of the proposed system for retractable roofs of medium to large span. The open and closed 
configurations were taken at $\beta_{\mathrm{f}}=30^{\circ}$ and $\beta_{\mathrm{uf}}=120^{\circ}$ corresponding to the actuation range of the PPAM.

\subsection{Approach to structural analysis}

In general, the membrane surface and the supporting structure of a tensile surface structure are analysed separately, with different calculation methods, models, and software tools. However, given the kinematic nature and flexibility of this retractable membrane structure, calculating the membrane and the supporting structure separately, without considering the effects of their interaction, would lead to an overestimation of the performance of the structure as a whole, ultimately leading to its failure, even when the results for the separate components were satisfactory.

Therefore, integrated models were used that include the scissor-hinged frames, the membrane surface, the cables, and the additional supporting structure over which the frames are cantilevered. The calculations on these integrated structures were performed with a combination of two software tools, EASY (TechNet Gmbh) and RStab (Dlubal Gmbh).

The EASY software suite provides tools for force-density controlled formfinding of tensile surfaces, and for the static analysis of these surfaces and - to a certain extent - of their supporting structure (EASY, 2007; Gründig et al., 2000). RStab is a conventional FEM-tool for the calculation and analysis of frame structures (RStab, 2008).

The procedure is as follows. First, the entire structure is modelled using the different modules of EASY (EASY-Form, EASY-San and EASY-Beam). Material and section properties can be imported into EASY-Beam, from the material and section libraries of RStab, and attributed to all elements of the structure. The distribution of forces throughout the structure is then calculated in EASY-Beam. Then, since EASY does not provide tools for stress or buckling analysis, the elements of the supporting structure and the calculated internal forces are exported to RStab. There, deformation of the structure, and stress levels and buckling characteristics of the individual elements are checked. Material and section properties can be changed, if necessary, and imported back into EASY-Beam where the force distribution is recalculated. This procedure is repeated until the behaviour of the structure and all of its components are satisfactory.

To reduce the complexity of the models and the calculations (and also, in a way, due to the limitations of the software), some aspects of the structure had to be simplified. For example, the actuators were not included; the scissor bars were not organised in separate layers; and, instead of the cable-pulley system, individual cable segments were used, similar to the situation in Figure 11.

\subsection{Loads and load combinations}

In Structural Design of Retractable Roof Structures (Ishii, 2000), it is suggested that retractable roofs should be closed under extreme weather conditions, even when this is not required for use of the facility below. Therefore, wind and snow loads and their combinations were calculated for the closed roof; and in accordance with the Eurocode (Eurocode 1, 2005) and the European Design Guide for Tensile Surface Structures (Mollaert and Forster, 2004). Only wind loads that result from wind action parallel to the transformation direction of the roof were considered. The resulting load combinations are summarised in Table 1. 


\subsection{Comparative study of individual scissor arches}

To determine the number of units to be used in the integrated model, we evaluated the behaviour of an individual arch as a function of the number of units it contains. We also evaluated the influence of the angle between the bars in the unfolded configuration, $\beta_{\mathrm{uf}}$, by comparing arches with $\beta_{\mathrm{uf}}=90^{\circ}$ and $\beta_{\mathrm{uf}}=120^{\circ}$. For this comparative study, the traditional approach to the calculation of membrane surfaces and their supporting structure was used, with separate models.

Table 1 Load combinations for ultimate and serviceability limit state analyses

\begin{tabular}{lc}
\hline Load case & Combination \\
\hline 1 & $C_{1} \times$ selfweight $(\mathrm{sw})$ \\
2 & $C_{1} \times \mathrm{sw}+C_{2} \times$ snow \\
3 & $C_{1} \times \mathrm{sw}+C_{2} \times$ snow $+0.6 C_{2} \times$ wind \\
4 & $C_{1} \times \mathrm{sw}+C_{2} \times$ snow (asymmetrical) \\
5 & $C_{1} \times \mathrm{sw}+C_{2} \times$ snow (asymmetrical) $+0.6 C_{2} \times$ wind \\
6 & $C_{1} \times \mathrm{sw}+C_{2} \times$ wind \\
7 & $C_{1} \times \mathrm{sw}+C_{2} \times$ wind $+0.5 C_{2} \times$ snow \\
8 & $C_{1} \times \mathrm{sw}+C_{2} \times$ wind $+0.5 C_{2} \times$ snow (asymmetrical) \\
\hline
\end{tabular}

Note: ULS $\Rightarrow C_{1}=1.35$ and $C_{2}=1.5$. SLS $\Rightarrow C_{1}=1.00$ and $C_{2}=1.00$.

The scissor arches were analysed in RStab as 2-dimensional structures. The loads on the scissor-arches were derived from the reactions at the supports of corresponding membrane surfaces generated with EASY. Each scissor arch was considered the middle of three arches that support two membrane strips. The width of the membrane strips (and consequently the distance between the parallel arches that support them) was taken identical for all arches, at $6.5 \mathrm{~m}$, regardless of the number of units. The membrane strips were subjected to the load cases from Table 1.

Strength and stability of the individual members were checked under ultimate limit state (ULS) conditions; and the deformation of the scissor arches under serviceability limit state (SLS) conditions, by evaluating the displacements of the nodes. The limit to these displacements was set at $1 / 250$ of the total span, which is $176 \mathrm{~mm}$. Only in-plane loads were considered and the global stability of the arches was not calculated. The scissor-arches were compared by their weight, stiffness and size of their members.

The cables play an important role in the behaviour of all structures. In general, the dimensions of the bars, and therefore often the total weight of the structure, could be reduced by increasing the size of the cables; they reduced bending in the bars by limiting the displacement of the top and bottom hinges. This effect was limited for structures where out-of-plane buckling of the scissor-bars was critical. Load case 1 (snow) and 6 (wind) were the critical load cases for all structures. The top cable segments and the zigzag-cable segments are mostly tensioned by the snow load, and the bottom segments by wind. In both cases this corresponds to the deformed shape of the structure. 
Figure 21 Calculation models: (top) membrane surface for calculations in EASY (bottom) model of scissor arches for calculations with RStab
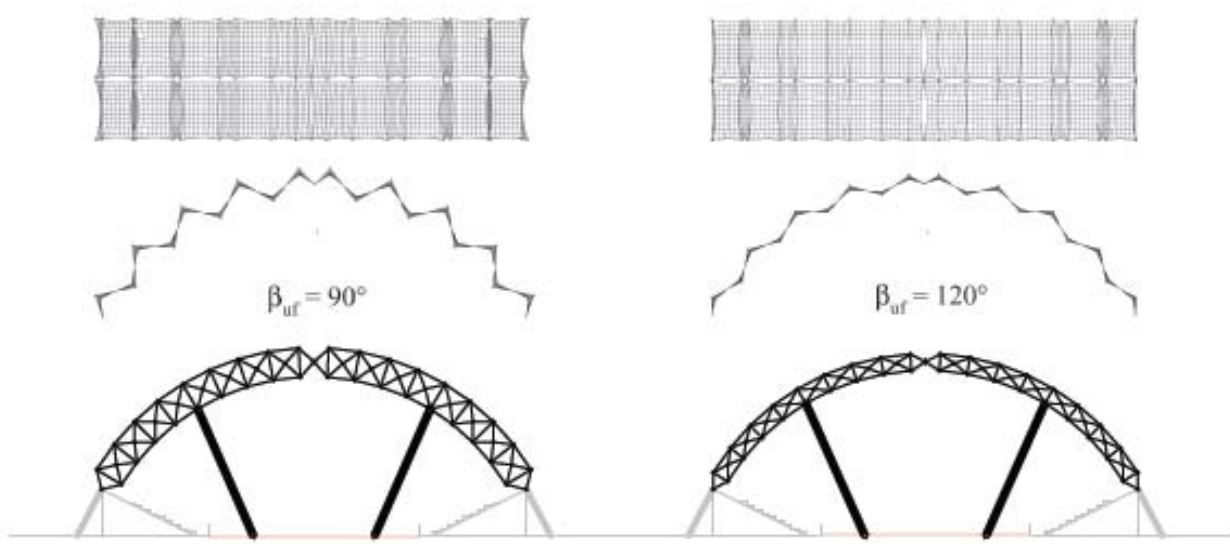

Figure 22 (a) Total weight of the structures and (b) maximum displacement of the nodes (see online version for colours)

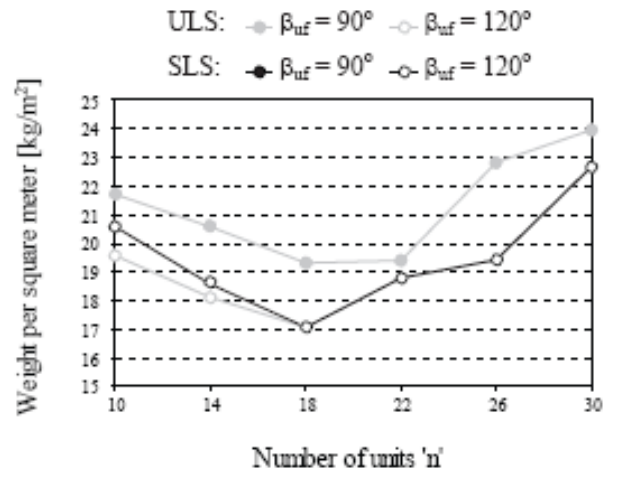

(a)

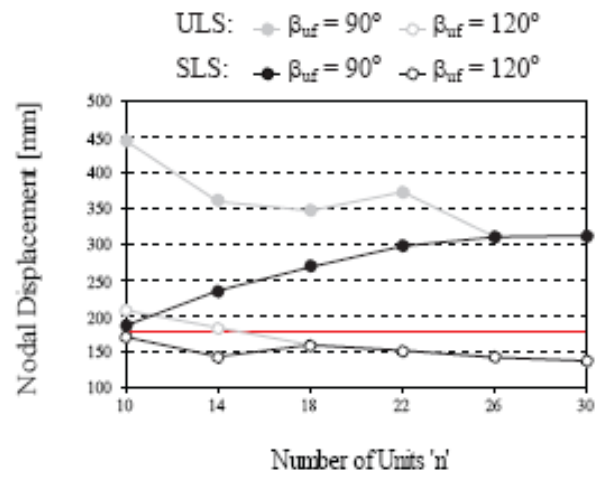

(b)

The structure with $\beta_{\mathrm{uf}}=120^{\circ}$ and $n=18$ is clearly the lightest solution (Figure 22), and requires the smallest member sizes (Table 2). This structure was chosen for further investigation with the 'integrated model'-approach. The deformation of the structure is highest at the central hinge, in the middle; the hinge is displaced by $-0.162 \mathrm{~m}$ under influence of snow, which is significantly lower than the displacements for the structures with $\beta_{\text {uf }}=90^{\circ}$ (Figure 22).

Evaluation of the deformation of the membrane surface showed that there was no possible drainage from several panels under snow loading (Figure 23). Since this problem is the result of the curved geometry of the roof - which causes some of the membrane panels to be almost horizontal - it could not be solved by choosing a different structure, for example $\beta_{\mathrm{uf}}=90^{\circ}$ and $n=18$, with more inclined membrane panels. 
Table 2 Overview of the total weight per element type and the total weight of the structure, after SLS calculations on the individual arches

\begin{tabular}{lccccc}
\hline$n$ & $\begin{array}{c}\text { Scissor-bars } \\
\text { section [mm] }\end{array}$ & Weight [kg] & $\begin{array}{c}\text { Cables } \\
\text { section [mm] }\end{array}$ & Weight [kg] & $\begin{array}{c}\text { Structure } \\
\text { weight [kg] }\end{array}$ \\
\hline \multicolumn{6}{c}{$\beta_{\text {uf }}=90^{\circ}$} \\
\hline 10 & $160 \times 6$ & 4432 & 28.6 & 725 & 6564 \\
\hline 10 & $160 \times 6.3$ & 3755 & 28.6 & 602 & 5659 \\
14 & $140 \times 6$ & 3086 & 28.6 & 633 & 5252 \\
18 & $120 \times 6$ & 2598 & 28.6 & 646 & 4780 \\
22 & $140 \times 6$ & 3072 & 28.6 & 657 & 5260 \\
26 & $150 \times 6.3$ & 3459 & 32.1 & 836 & 5625 \\
30 & $150 \times 6.3$ & 3457 & 36.6 & 1097 & 6084 \\
\hline
\end{tabular}

Therefore, a variation on the ridge-and-valley configuration with 'arching' compression elements at the ridges - instead of 'hanging' cables - was chosen for further investigation in the integrated model. This surface has a more pronounced doubly curved shape, and, if the upright position of the arches is guaranteed by an appropriate design of the connection with the scissors, ponding could be avoided with it (Figure 23).

Figure 23 Deformation of the membrane surface of the structure with $\beta_{\mathrm{uf}}=120^{\circ}$ and $n=18$; (a) ridge-and-valley surface, (b) ridge-and-valley surface with arching ridges (see online version for colours)

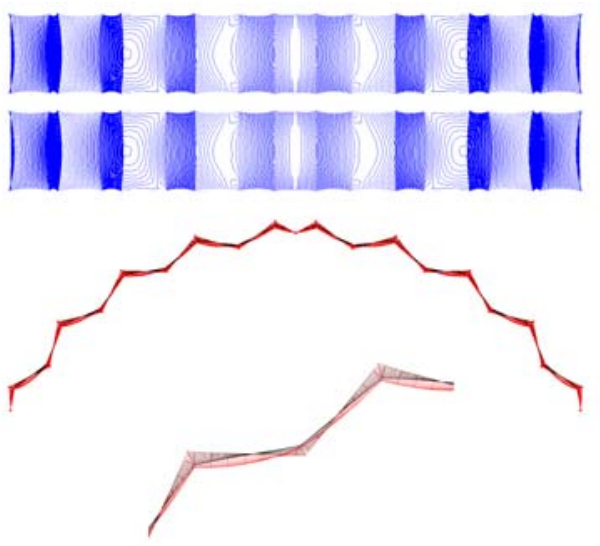

(a)
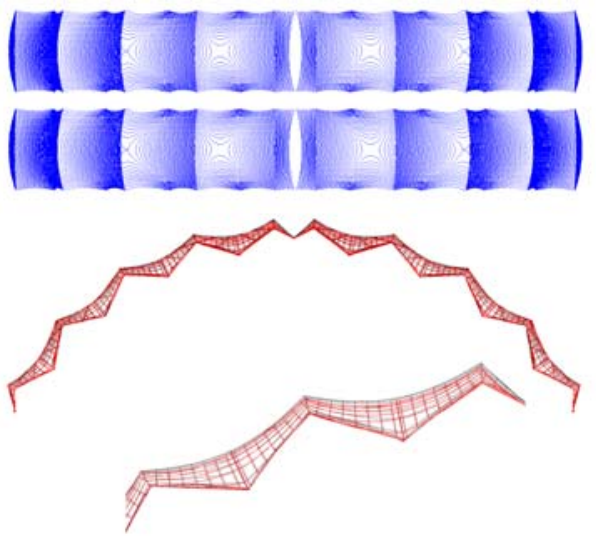

(b)

\subsection{Integrated model}

The integrated calculation model is similar to the model discussed in Subsection 4.2; except for the fact that the ridge cables are replaced by arching compression ridges, and that the PPAM are not included (Figure 24). 
Figure 24 Integrated model of two strips of the entire roof; ( $a$ and $b$ ) closed configuration of the roof (c) cantilevered, completely unfolded, individual frame (see online version for colours)

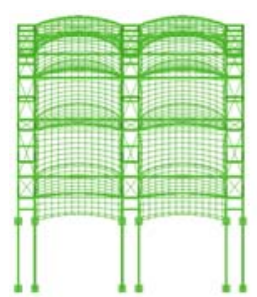

(a)

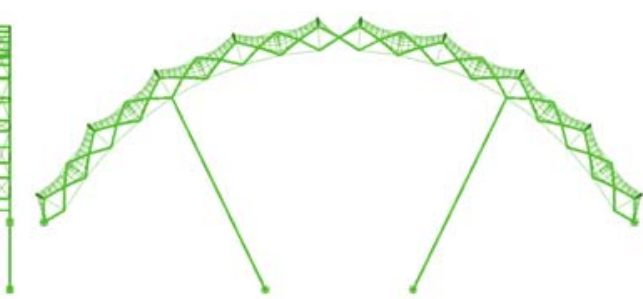

(b)

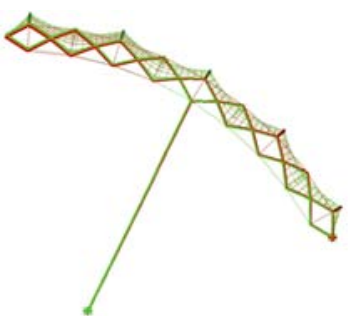

(c)

The model is calculated with EASY-Beam and RStab as discussed in Subsection 5.1. The sections that were found for the structure with $\beta_{\mathrm{uf}}=120^{\circ}$ and $n=18$ through the analysis of the individual arches (Table 2) were also used for the bars and cables of this model. Since in this case a double layer of scissors was used, instead of a single layer as for the individual arches, in many elements only a fraction of their strength and buckling capacity was used. However, attempts at reducing these sections resulted in instabilities in the model, which demonstrates the difference between calculations with separate or integrated models. The total weight of the two strips per square metre of covered surface are is $35,575 \mathrm{~kg} / 1,358.5 \mathrm{~m}^{2}=26.2 \mathrm{~kg} / \mathrm{m}^{2}$. With more detailed calculations, where the elements are designed individually, rather then by type, it should be possible to reduce the weight of the structure even further, especially at the tip of the frames, in the middle of the closed roof.

Lastly, some calculations were performed on a completely unfolded, individual frame. Only the selfweight of the structure was considered during these calculations. The displacement of the central hinge in this configuration, at the tip of the frame, is $-0.182 \mathrm{~m}$. This is not only more than the limit of $0.176 \mathrm{~m}$, it also complicates the way in which the frames should be connected at that hinge to close the roof. A reduction of the weight of the tip of the frame, as discussed above, as well as the use of a multi-angulated mechanism could reduce this deformation.

\section{Conclusions}

The 'Scissor-hinged retractable membrane structures'-system is an interesting solution for retractable (membrane) roofs that require a fully retractable supporting structure.

Using cables and pulleys, the transformation of the supporting structure can be controlled with a minimum of motors, and in configurations where the structure is required to carry loads, the cables improve the structural behaviour of the scissor mechanisms significantly, by reducing bending in the bars. However, since the cable-pulley system was replaced by individual segments in the calculation models, the positive effect of the cables may have been overestimated. More detailed calculations and models are required to evaluate the behaviour of a scissor-hinged cable-pulley system correctly. 
In an attempt to create a membrane roof with multiple roof configurations, a ridge-and-valley membrane surface was spanned in the supporting structure, and actuators (PPAM) were integrated to control the tension in the membrane as the roof transforms from one configuration to another. The feasibility of this principle - although demonstrated on a small membrane module - will largely depend on the feasibility of the detailing that is required to create a fully closed roof surface. For example, at this point, the borders of the membrane that run alongside the scissor arches are not yet connected to the scissor bars. This connection should be sufficiently flexible to allow for the actuation of the valley cables by the PPAM; maybe patches of stretchable fabric could be used. Furthermore, along the way, some changes had to be made to the configuration of the membrane that may compromise the 'foldability' of the surface. Of course, most of these problems may be solved by simply replacing the membranes with inflatable cushions or even simple rigid panels.

Further research should focus on the development of specialised software, in which the (inter)action of all elements - in specific configurations and during transformation can be modelled more realistically; and on the construction of detailed physical models, in which the foldability of different types of ridge-and-valley surfaces and the transformation of the supporting structure with the cable-pulley system can be tested.

\section{References}

Block, P. and Van Mele, T. (2003) 'Scissor-hinged deployable membrane structures, tensioned by pleated pneumatic artificial muscles', Master thesis, Vrije Universiteit Brussel, Brussels, Belgium.

Daerden, F. and Lefeber, D. (2002) 'Pneumatic artificial muscles: actuators for robotics and automation', European Journal for Mechanical and Environmental Engineering, Vol. 47, No. 1, pp.11-22.

De Temmerman, N. (2007) 'Design and analysis of deployable bar structures for mobile architectural applications', Phd thesis, Vrije Universiteit Brussel, Brussels, Belgium.

EASY (2007) User Manual for Easy, TechNet Gmbh.

Eurocode 1 (2005) 'Actions on structures', Technical Report EN 1991, European Committee for Standardisation.

Gründig, L., Moncrieff, E., Singer, P. and Ströbel, D. (2000) 'A history of the principal developments and applications of the force density method in Germany 1970-1999', in IASS-IACM 2000 Fourth International Colloquium on Computation of Shell and Spatial Structures, 5-7 June, Chania-Crete, Greece.

Ishii, K. (2000) Structural Design of Retractable Roof Structures, WIT Press, Ashurst, Southampton, UK.

Jensen, F. (2004) 'Concepts for retractable roof structures', Phd thesis, University of Cambridge, Cambridge, UK.

Mollaert, M. and Forster, B. (Eds.) (2004) European Design Guide for Tensile Surface Structures, 1 st ed., VUB Press, Brussels, Belgium.

RStab (2008) User Manual for RStab 6, Dlubal.

Van Mele, T. (2008) 'Scissor-hinged membrane structures - a novel system for retractable membrane roofs', Phd thesis, Vrije Universiteit Brussel, Brussels, Belgium.

You, Z. and Pellegrino, S. (1997) 'Foldable bar structures', International Journal of Solids and Structures, Vol. 1, No. 34, pp.1825-1847. 


\section{Notes}

$1 \quad M$ is measured along a straight line between the cables. Of course, $M$ should actually be measured over the surface. However, since a ridge-and-valley surface typically has only slightly warped panels, a straight line is a good approximation. 